\title{
EDITORIAL
}

\section{Introduction to the special issue of the 2009 International Symposium on Crystal Engineering \& Drug Delivery Systems}

\author{
Zhijian CHEN* $(\bowtie)$, Wei LI* ${ }^{*}()$ \\ School of Chemical Engineering and Technology, Tianjin University, Tianjin 300072, China
}

(C) Higher Education Press and Springer-Verlag Berlin Heidelberg 2010

On September 5-8, 2009, the 2009 International Symposium on Crystal Engineering \& Drug Delivery Systems was held in Tianjin, China. This conference was in succession to the 1998 and 2004 International Symposium on Industrial Crystallization that were held in Tianjin and organized by the State Research Center for Industrialization of Crystallization Technology (SRCICT) at Tianjin University.

The aim of the conference was to encourage the presentation and discussion of the latest advances in theories and applications broadly in the fields of crystal engineering and drug delivery systems. In addition, this conference intended to strengthen the network and communications among academic and industrial researchers. Ten topics were discussed in the conference, including advances in R\&D for industrial crystallization technology, pharmaceutical crystallization technology, recent developments in nanocrystals and biomacromolecule crystals, design and control of industrial crystallization processes, molecular modeling in crystal engineering, modern pharmaceutics and drug delivery systems, modern Chinese herbal medicine, tissue engineering and cell delivery, biomaterials and drug carriers, and new drug design, synthesis and modification. The three-day conference featured a program composed of four plenary lectures, 20 specially invited lectures, 30 invited lectures, and a poster section. The participants were from USA, Germany, Netherland, Canada, Spain, Singapore, Japan, Korea, and China. This special issue of Frontiers of Chemical Engineering in China contains a representative selection of papers from more than 120 contributions covering the above-mentioned topics. All the papers were subject to the peer review process of the journal. These reviews and research articles cover a wide spectrum of interesting topics such as pharmaceutical crystallization, crystal polymorphism, nanocrystals and nanomaterials, crystallization in microfluidic devices, crystallization modeling, macromolecular therapeutics, drug delivery carriers, active components analysis of Chinese herbal medicines, and enzyme immobilization for drug synthesis. It is our hope that this special issue provide the readers with valuable insights into current researches on industrial crystallization and drug delivery systems.

We thank all the authors and referees for their commendable support. We would also like to express our sincere appreciation to Prof. Jingkang Wang, the Editor-in-Chief, and Dr. Fei Yuan and Prof. Yaodong Huang, the Managing Editors of this journal, for their advices and efforts to develop this special issue.

E-mail: zjchen@tju.edu.cn, liwei@tju.edu.cn

*Prof. Zhijian CHEN and Prof. Wei LI are the guest editors for the special issue of the 2009 International Symposium on Crystal Engineering \& Drug Delivery Systems 